\section{Facilitadores del reintegro laboral en trabajadores con patología mental de origen laboral: una revisión sistemática}

\author{
ELISA ANSOLEAGA ${ }^{1,2, a}$, PABLO GARRIDO ${ }^{3,4, b}$, \\ CONSTANZA DOMÍNGUEZ ${ }^{1, \mathrm{c}}$, SOLEDAD CASTILLO ${ }^{1, \mathrm{c}}$, \\ CLAUDIA LUCERO ${ }^{2,5, \mathrm{~d}}$, ALEMKA TOMICIC ${ }^{6,7, \mathrm{e}}$, \\ CLAUDIO MARTÍNEZ ${ }^{2,6,7, \mathrm{e}}$
}

\section{Return to work enablers for workers with work-related mental illness}

\begin{abstract}
Background: There is growing interest in the treatment and return-to-work of workers with labor related mental illnesses. Aim: To perform a systematic review of practices and interventions that improve return to work. Material and Methods: Systematic literature review. Thirty articles were selected for in-depth analysis. Results: Self efficacy perception, work motivation, a lower age and a better socioeconomic status were identified as worker-related return to work facilitators. Among work environment facilitators, good communication practices, supervisor support, a good assessment and modification of work load, adjustment of expectations, a good relationship between employers and employees and positive work experiences were identified. Treatment may improve return to work using a multidisciplinary approach, reducing stress and identifying psychosocial determinants of mental problems rather than symptoms and providing a timely health care. Conclusions: Return to work of workers with labor related mental illnesses requires a constant sharing of information between health care workers, employers and employees to identify common therapeutic objectives.

(Rev Med Chile 2015; 143: 85-95)
\end{abstract}

Key words: Mental Disorders; Mental Health; Occupational Health; Return to Work.

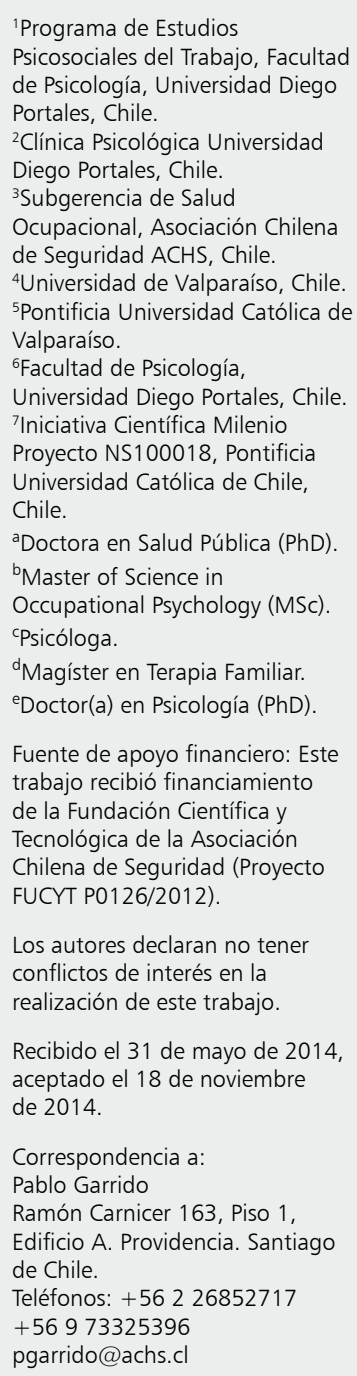

Los autores declaran no tener conflictos de interés en la realización de este trabajo.

Recibido el 31 de mayo de 2014 aceptado el 18 de noviembre de 2014.

Correspondencia a:

Pablo Garrido

Ramón Carnicer 163, Piso 1 ,

Edificio A. Providencia. Santiago

de Chile.

Teléfonos: +56 226852717

+56973325396

pgarrido@achs.cl

E n la actualidad, según la Organización Mundial de la Salud (OMS), la salud ocupacional abarca la búsqueda de bienestar físico y mental de los trabajadores, generado a partir de las condiciones laborales que permitan un entorno saludable. Esta búsqueda de condiciones para lograr el bienestar mental tiene correlato en la generación creciente de estudios sobre los llamados aspectos psicosociales del trabajo ${ }^{1}$, que buscan su identificación, aumento de la identificación de patologías y aumento de licencias médicas asociadas a enfermedades de carácter mental.

El tratamiento y reintegro laboral de personas diagnosticadas con patologías mentales relacionadas con el trabajo revisten un creciente interés y escaso desarrollo en la región. La mayor cantidad de publicaciones científicas en este tema se concentra en los últimos 3 años y no se encontraron publicaciones de Chile e Iberoamérica ${ }^{2}$. Según la $\mathrm{OPS}^{3}$, la carga de enfermedad debida a trastornos mentales y neurológicos representa 22\%, y sólo alrededor de $45 \%$ de los países panamericanos miembros refieren contar con una política nacional de salud mental. Chile, aun cuando cuenta con un plan nacional de salud mental y psiquiatría desde 1993, no dispone de un marco legal y de 
políticas específicas para enfrentar las patologías del ámbito de la salud mental ocupacional. Si bien la legislación chilena de protección de la salud en el trabajo (Ley 16.744) incluye desde sus orígenes la llamada 'neurosis laboral', , se mantiene el concepto de 'excesiva tensión psíquica' como el agente de riesgo desencadenante, siendo difícil determinar a qué se refiere este concepto en el ámbito laboral. Tal definición no es trivial, dado que determinará qué sistema de seguro (ocupacional o común) se hará cargo del tratamiento y rehabilitación del trabajador enfermo, con los diversos costos y efectos que aquella decisión conlleva ${ }^{5}$. En este contexto, la inclusión de distintos enfoques que permitan acceder a la complejidad que supone proteger el reintegro laboral de una persona al espacio donde construye identidad, pasa gran parte de su tiempo, y también requiere desarrollarse, es una recomendación en este ámbito ${ }^{6}$.

En la esfera de la salud mental ocupacional, el panorama latinoamericano continúa siendo incipiente; sólo Chile y Colombia cuentan con protocolos de vigilancia epidemiológicos para los riesgos psicosociales del trabajo ${ }^{7-8}$, los cuales intervienen a nivel de diagnóstico del riesgo ambiental, pero aún carecen de protocolos específicos para la intervención y prevención de los elementos desencadenantes del estrés laboral.

Considerando que el gasto e incapacidad temporal generada por las patologías de salud mental en algunos de los países de las Américas supera los 50 billones de dólares anuales ${ }^{9}$, que el gasto público en salud mental en Chile no llega al 3\% comparado con el $10 \%$ promedio de países de la $\mathrm{OECD}^{10}$, que la mayor concentración de patología mental de origen laboral se produce en edades productivas $^{11}$, y que las causas de dicha incapacidad no se explican sólo por la condición clínica del paciente sino por un conjunto de factores sociolaborales que vinculan la relación del empleador con el trabajador y el tratante ${ }^{2}$ y son responsables de más de $65 \%$ de los determinantes no-clínicos de la enfermedad ${ }^{12}$, es que el proceso de reintegro laboral y/o las prácticas que producen un reintegro exitoso, deben ser un tema de creciente interés.

Revisiones sistemáticas y meta-análisis han descrito facilitadores y obstaculizadores asociados al retorno al trabajo, abordando indistintamente problemas de salud mental y afecciones generales de salud. Ellos destacan la necesidad de mantener comunicación entre supervisor y empleado ${ }^{13}$, realización de intervenciones tempranas, multidisciplinarias $^{14-15}$, y que integran el lugar de trabajo e intervenciones focalizadas en desarrollar estrategias de afrontamiento en el paciente ${ }^{16}$, entre otras.

Este trabajo identifica, a través de una revisión sistemática de la literatura de los últimos cinco años, aquellas prácticas e intervenciones que han resultado exitosas para el reintegro al trabajo en personas con problemas de salud mental laboral.

\section{Material y Método}

Se realizó una revisión sistemática de la literatura científica, con un enfoque cualitativo, siguiendo las orientaciones de la declaración PRISMA ${ }^{17}$. Las bases de datos utilizadas para la búsqueda fueron: Pubmed, Psychology and Behavioral Sciences Collection (EBSCOhost), MEDLINE con texto completo (EBSCOhost), Academic Search Complete (EBSCO host), MEDLINE (EBSCO host), ProQuest Psychology Journals, PsycINFO (EBSCOhost) y PsycArticles (EBSCOhost).

Se emplearon los siguientes términos de búsqueda y combinaciones de los mismos: "return to work", "mental disorder", "cognitive-behavioral therapy", "burnout”, "occupational health", "mental depression", "psychotherapy", "sickness absence", "work stress", "workplace", "work environment" y "treatment outcome". Se utilizaron los mismos términos en español, pero no se encontraron publicaciones. El criterio temporal de búsqueda correspondió a los últimos 5 años (2008 a 2012). En el período de búsqueda de diciembre de 2012 a marzo de 2013, se encontraron 142.759 artículos.

El tamizaje de los artículos se realizó considerando los siguientes criterios de inclusión: a) que abordaran problemas de salud mental; b) que consideraran el retorno al trabajo y c) que fueran artículos de publicaciones indexadas en sistemas ISI y/o Scielo. Los criterios de exclusión fueron: a) artículos de revisiones sistemáticas o meta-análisis, por la imposibilidad de comparar el alcance de sus resultados con los de los artículos de investigación incluidos en esta revisión; b) artículos teóricos o conceptuales; c) artículos en que se presentaron diseños de intervención, pero no su aplicación y/o resultados y d) artículos referidos al retorno al trabajo de personas con enfermedades físicas y que consideraran sólo de forma secundaria problemas de salud mental. 


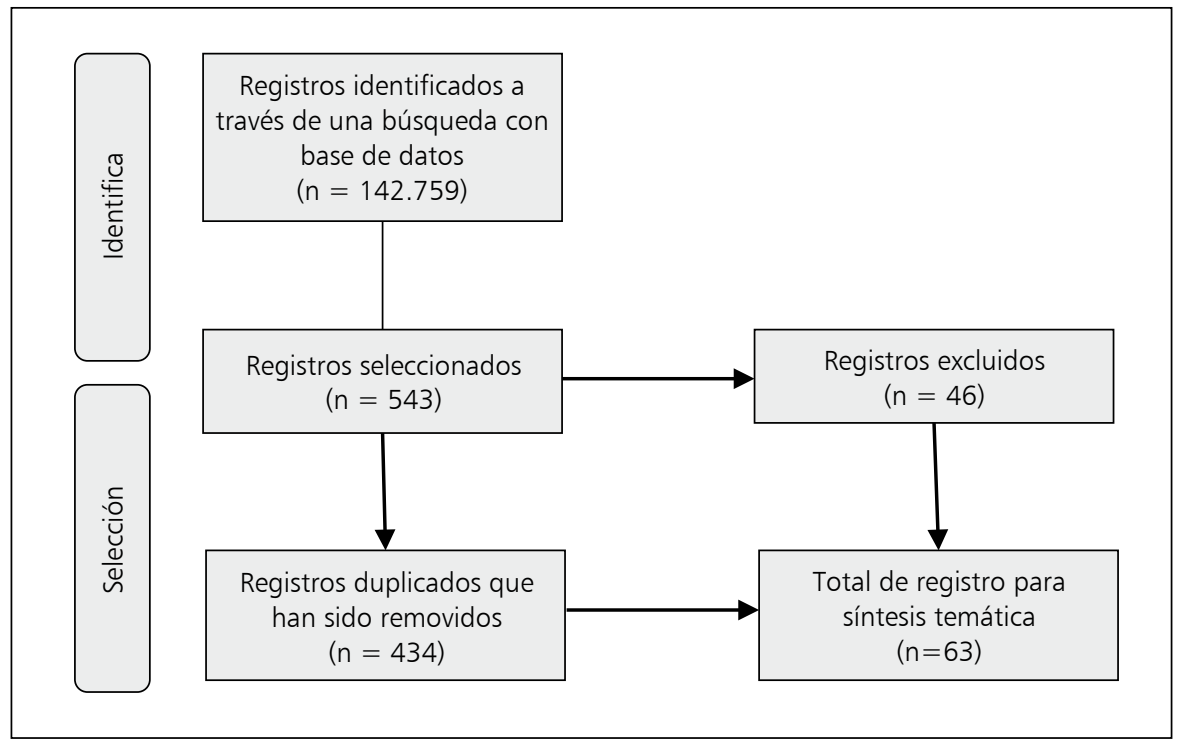

Figura 1. Diagrama de flujo que ilustra el proceso de inclusión y exclusión de los registros.
La Figura 1 grafica el proceso de búsqueda, selección, inclusión y exclusión de los registros. $\mathrm{Al}$ finalizar este proceso se seleccionaron 63 artículos para el proceso de revisión.

\section{Procedimiento de revisión}

Se construyó una base de datos para el análisis temático de cada uno de los artículos seleccionados, definiéndose quince categorías, las que posteriormente fueron recodificadas en siete categorías finales (Tabla 1).
Para la identificación de factores de éxito o fracaso del reintegro laboral se seleccionaron 30 artículos que consideraron en sus resultados el proceso de reintegro laboral (Tabla 2).

\section{Resultados}

Los resultados se presentan en función de tres grandes categorías: a) características del trabajador; b) del ambiente de trabajo, y c) del tratamiento recibido.

\section{Tabla 1. Definición de categorías para análisis}

\begin{tabular}{|c|c|}
\hline Nombre de la categoría & Definición \\
\hline Año & Año de publicación del artículo \\
\hline Revista & Revista en que fue publicado el artículo \\
\hline País & País de procedencia del estudio \\
\hline Variable resultado & $\begin{array}{l}\text { Corresponde a la variable dependiente en un estudio experimental o cuasi-experimental. } \\
\text { Pero en términos más generales, aquí hace referencia a aquella variable que es objeto de } \\
\text { estudio o análisis }\end{array}$ \\
\hline Participantes & $\begin{array}{l}\text { Característica de la muestra y tamaño muestral: n total, sexo, edad, área laboral, tipo de } \\
\text { licencia }\end{array}$ \\
\hline Diseño & $\begin{array}{l}\text { Diseño y tipo de estudio. Si la intervención es con caso clínico controlado o no; retrospec- } \\
\text { tivo o prospectivo; transversal o longitudinal; cualitativo, cuantitativo o mixto }\end{array}$ \\
\hline $\begin{array}{l}\text { Instrumentos o dispositivos de } \\
\text { observación }\end{array}$ & $\begin{array}{l}\text { Técnicas e instrumentos de recolección de datos: técnicas cualitativas; auto reporte u ob- } \\
\text { servación; instrumentos estandarizados }\end{array}$ \\
\hline
\end{tabular}




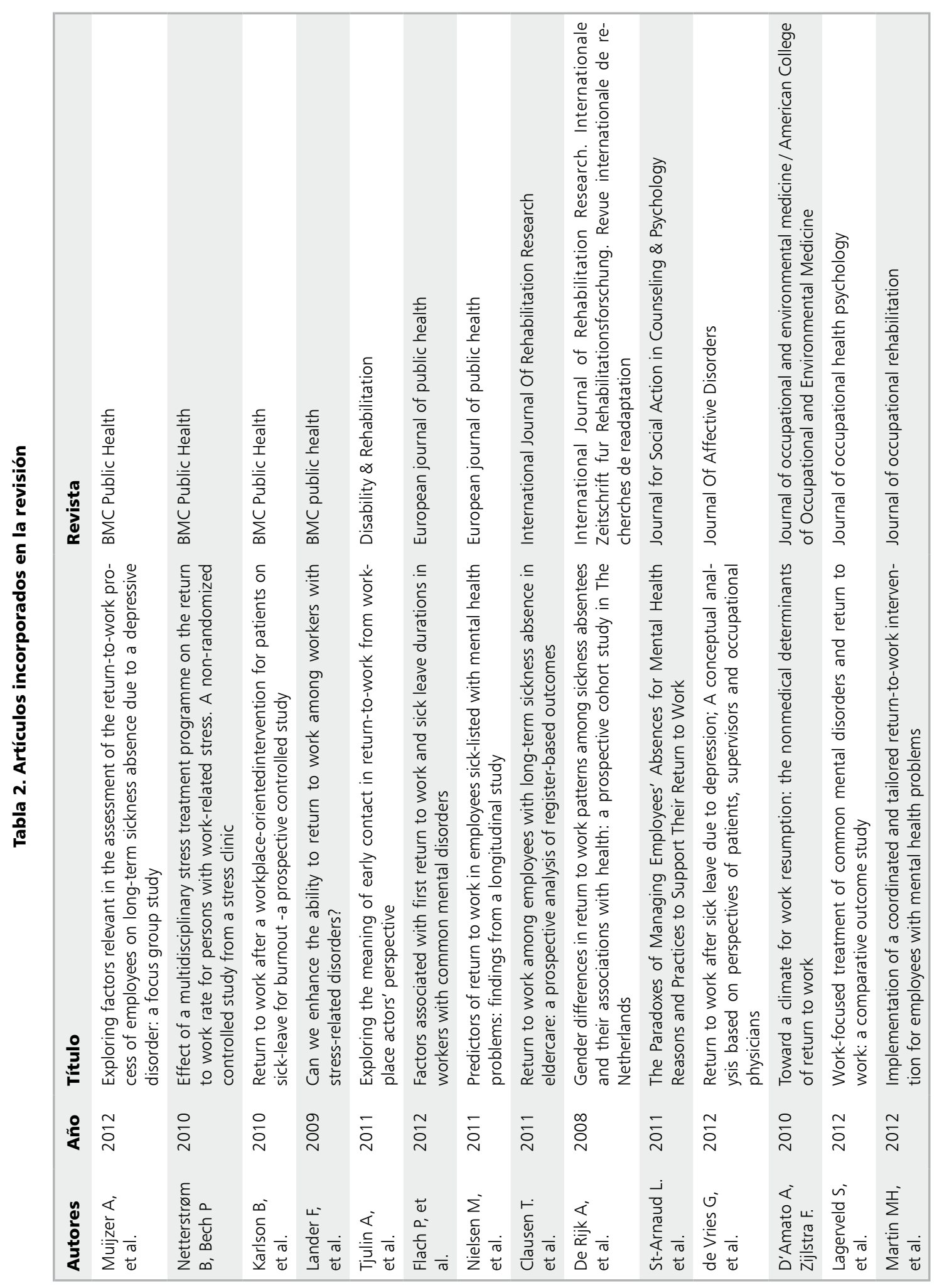




\begin{tabular}{|c|c|c|c|c|c|c|c|c|c|c|c|c|c|c|c|}
\hline 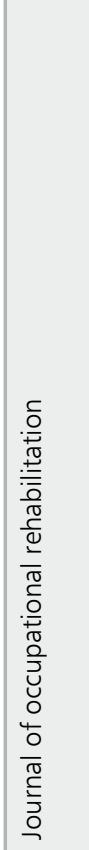 & 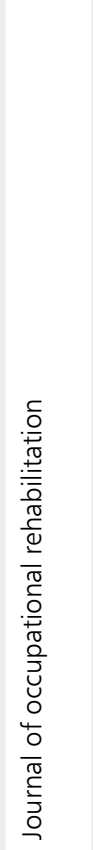 & 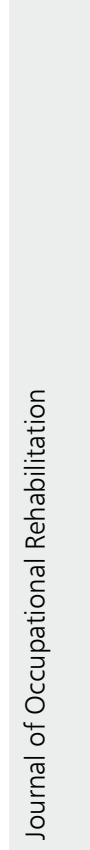 & 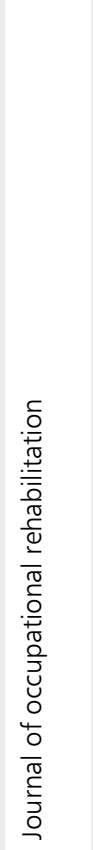 & 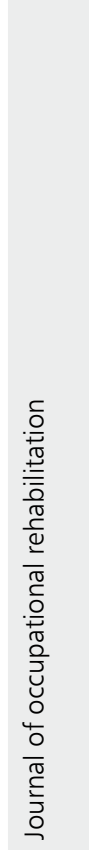 & 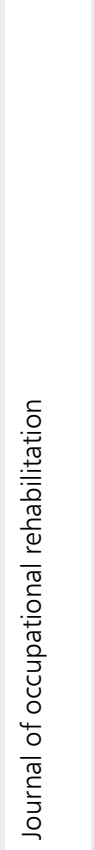 & 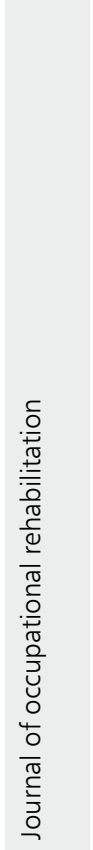 & 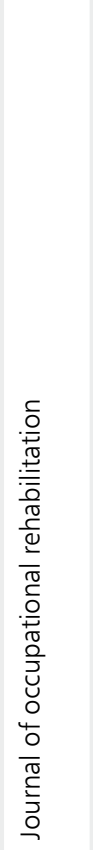 & 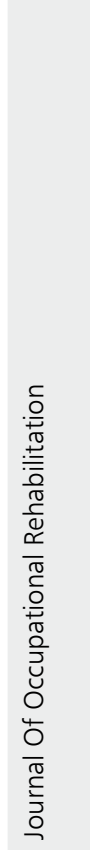 & 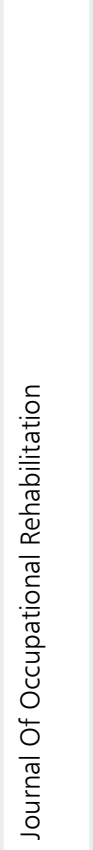 & 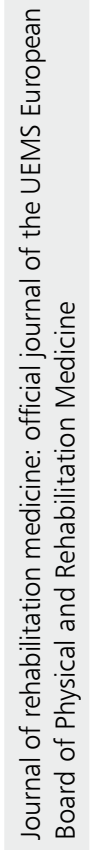 & 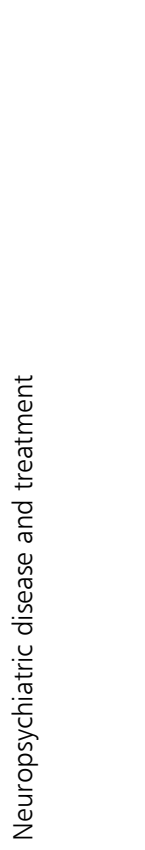 & 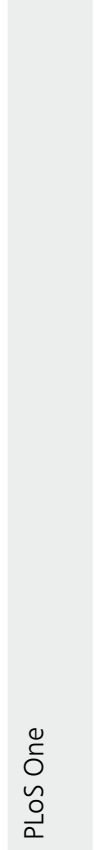 & 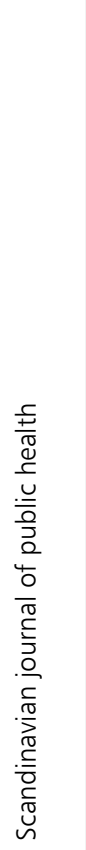 & 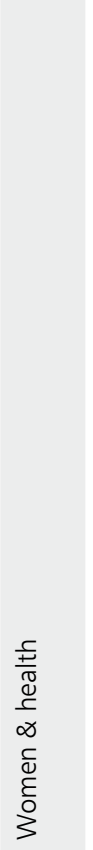 & 蒿 \\
\hline 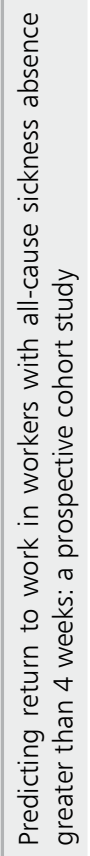 & 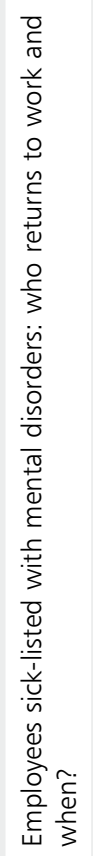 & 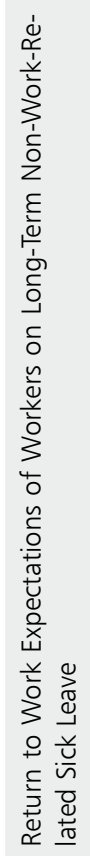 & 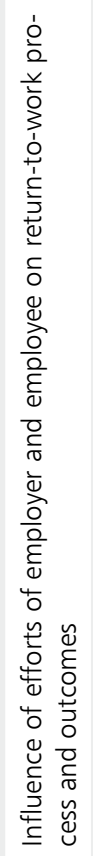 & 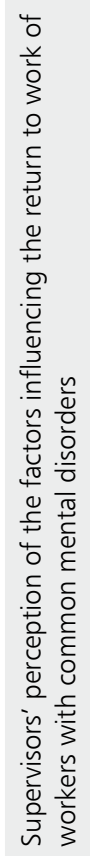 & 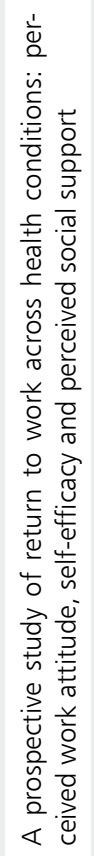 & 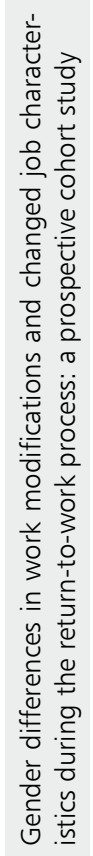 & 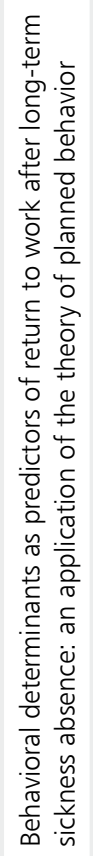 & 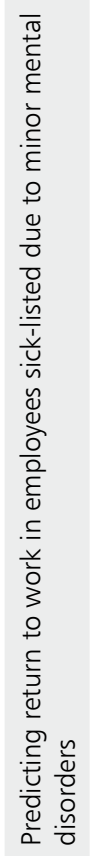 & 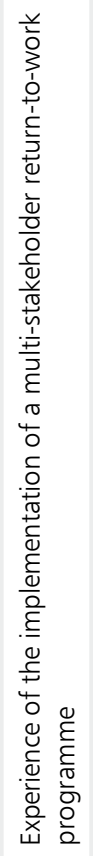 & 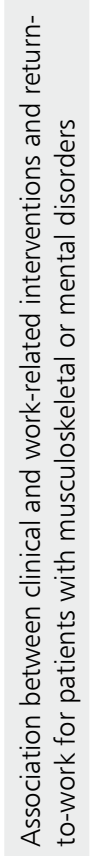 & 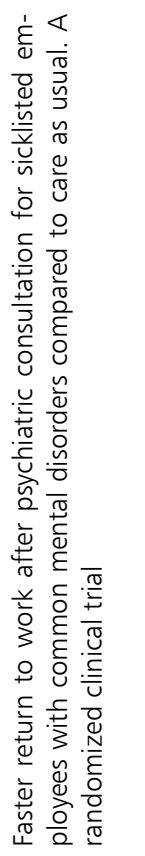 & 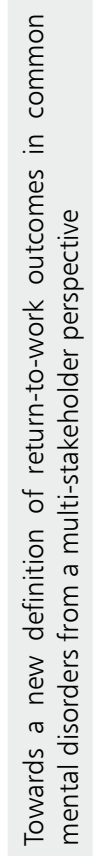 & 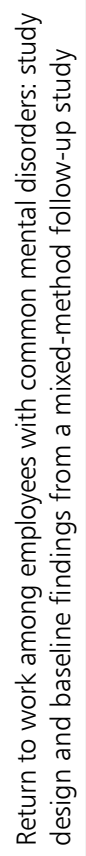 & 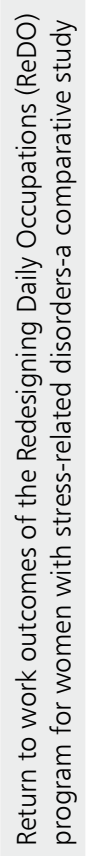 & 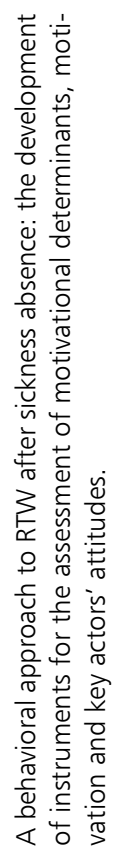 \\
\hline$\stackrel{\sim}{\stackrel{N}{\sim}}$ & $\stackrel{\sim}{\sim}$ & $\stackrel{\sim}{i}$ & $\bar{i}$ & $\overline{\bar{N}}$ & $\stackrel{\circ}{\stackrel{D}{\nu}}$ & ᄋ̊̀ & ஜे & ஜे & ஜे & $\stackrel{\sim}{\sim}$ & $\stackrel{\circ}{\frac{N}{\sim}}$ & $\stackrel{\sim}{\stackrel{\sim}{\sim}}$ & $\stackrel{\circ}{\stackrel{N}{N}}$ & $\bar{i}$ & 오 \\
\hline 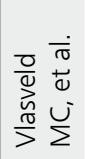 & 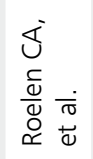 & 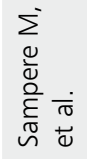 & 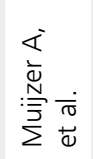 & 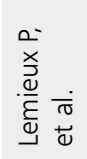 & 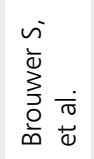 & 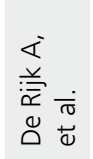 & 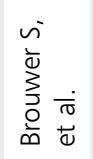 & 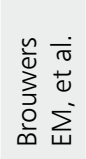 & 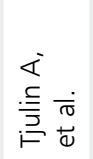 & 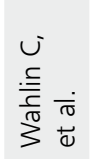 & 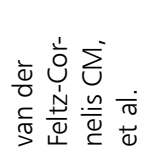 & 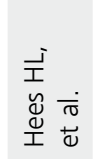 & 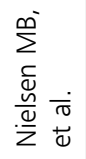 & 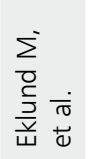 & 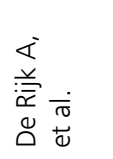 \\
\hline
\end{tabular}


Facilitadores para el reintegro laboral en personas con patología mental - E. Ansoleaga et al

\section{a) Aspectos relacionados con el/la trabajador/a en reposo por incapacidad}

Entre los elementos individuales que se señalan como facilitadores para el reintegro al trabajo destacan una alta percepción de autoeficacia del trabajador ${ }^{18-21}$. Así, la visión que tenga el trabajador con respecto al aporte de su labor determinará el progreso de su reintegro al trabajo ${ }^{19-22}$. Por otra parte, Clausen y col. ${ }^{23}$, plantean que la motivación del trabajo es un recurso para el reintegro, pues refuerza la voluntad de los empleados de volver al trabajo después de largos períodos de ausencia por enfermedad ${ }^{20,21,24}$. La percepción de un adecuado estado de salud por parte del trabajador ${ }^{19-20}$ contribuirá al proceso de reintegro y, por el contrario, la percepción de una salud deteriorada lo retardará. Ahora bien, cuando la patología mental se ha ocasionado producto del burnout por el sobre-compromiso con el trabajo, el apoyo del supervisor será más significativo: a mayor apoyo del supervisor, mayor tasa de reintegro ${ }^{20}$.

Una vez que el trabajador ha retornado, la evidencia enfatiza que la disponibilidad de estrategias para que pueda afrontar y resolver los problemas propios del trabajo ${ }^{25}$ y la capacidad para reconocer tempranamente las señales de estrés laboral ${ }^{26}$ son factores de protección. En relación con procesos de reposo previo y/o recaídas, se refiere que la ausencia de recaída, o bien, la ausencia en el trabajador de la experiencia subjetiva de estar proclive a una recaída, facilitarían el proceso de reintegro ${ }^{22}$.

Respecto a factores sociodemográficos, los trabajadores de menor edad y de niveles socioeconómicos bajos se reintegrarían a sus quehaceres laborales de modo más rápido al comparárseles con trabajadores de mayor edad y de niveles socioeconómicos altos ${ }^{27}$. Trabajadores de más de 45 años tendrían mayores dificultades para retornar al trabajo, sin embargo, la relación entre edad y tiempo de retorno al trabajo es menos pronunciada y más inespecífica en las patologías mentales ${ }^{19}$. Por otro lado, resulta importante identificar particularidades del proceso de reintegro laboral según género ${ }^{28}$. Por ejemplo, en ambos sexos son comunes las readecuaciones en horario y ritmo de trabajo durante el proceso de reintegro al trabajo, sin embargo, estos son más frecuentes en mujeres que en hombres. Al retornar, aquellas mujeres que utilizaron el beneficio de readecuación percibieron mayor satisfacción laboral que aquellos hombres que no la utilizaron, los cuales percibieron una alta carga emocional post-retorno. Se sugiere que las diferencias en el trabajo asociadas al género podrían afectar el proceso de reintegro.

\section{b) Aspectos asociados al ambiente de trabajo}

Una adecuada comunicación entre los distintos actores involucrados y un diálogo abierto y franco entre el supervisor directo y el trabajador disminuye los sentimientos y cogniciones negativas del trabajador respecto a su lugar de trabajo $^{19,29}$, siendo relevante los acuerdos para mejorar y regular la comunicación ${ }^{26,30}$.

Otro elemento facilitador es que trabajador y empleador consideren que el primero está en condiciones de iniciar su reintegro laboral ${ }^{26}$. Se enfatiza la importancia que la jefatura confíe en que el empleado será capaz de manejar la carga de trabajo al momento del retorno ${ }^{26}$, asignando nuevamente las tareas que el trabajador tenía, e informando los cambios producidos durante su ausencia $^{31}$. Para esto, se debe considerar el ajuste entre las expectativas del trabajador y las del supervisor respecto al desempeño del primero durante el reintegro, destacando que la actitud del supervisor directo debiera caracterizarse por la apertura, flexibilidad, atención, empatía y apoyo ${ }^{31}$.

Los hallazgos respecto a la importancia del contacto precoz entre trabajador y empleador no son consistentes. Algunas investigaciones plantean que el contacto temprano entre el empleador y el trabajador no actúa siempre como un facilitador para el proceso de reintegro ${ }^{32}$. Sin embargo, también se enfatiza que lo positivo es mantener una actitud de apoyo del empleador hacia el trabajador para cuidar la relación entre ambos y promover el reintegro ${ }^{19,33}$. Finalmente, destaca la importancia de organizar el reintegro y, en tal sentido, la necesidad de reunirse con el trabajador desde el primer día de reintegro a su jornada laboral ${ }^{31}$.

Respecto a las tareas del trabajador, se considera relevante realizar las adecuaciones o modificaciones necesarias en las tareas-actividades ${ }^{34}$. Destaca, como parte de un proceso de intervención específico para el reintegro laboral, la reducción de la presión en el trabajo, el ritmo y las exigencias en general ${ }^{35}$. De Vries plantea tres importantes meta-clústers (trabajo, persona y tratamiento) para un adecuado tratamiento orientado al reintegro en administrativos y oficinistas. Respecto al trabajo, las intervenciones debieran poner énfasis en: a) adaptación del puesto de trabajo; b) comprensión 
y apoyo en el trabajo y c) procurar experiencias positivas de trabajo. Respecto a la persona, las intervenciones debieran enfocarse en: a) una autopercepción positiva del trabajador; b) mejorar competencias de autogestión; c) estimular un nivel positivo de energía física y ánimo hacia las tareas encomendadas y d) estimular un ambiente de apoyo en el hogar. Respecto al tratamiento, más allá de un tratamiento correcto del punto de vista de los medicamentos, psiquiatra/psicoterapia o terapia ocupacional, es la coordinación entre los distintos actores del proceso (empleador-pacienteequipo tratante-familia-otros), y el apoyo y guía percibidos por el paciente lo que hace más efectivo y eficiente el proceso de reintegro laboral ${ }^{18}$.

Un elemento a destacar es el apoyo y comprensión percibido por los trabajadores durante su proceso de reintegro ${ }^{18}$, y un adecuado nivel de apoyo social ${ }^{21}$ entregado por parte de los superiores y/o jefaturas hacia el trabajador reintegrado que incluya interés activo por la situación global del trabajador ${ }^{20}$. Netterstrom y Bech $^{34}$ plantean que el apoyo competente del supervisor facilita el desarrollo de actividades por parte del trabajador. Para que esto tenga efecto, es importante que los supervisores sean consistentes en sus demandas de reintegro, y que éstas se alineen con las políticas de la empresa. Se requiere un marco de referencia que defina el enfoque desde el cual se abordará el proceso de reintegro, siendo más conveniente un enfoque de apoyo-racional, es decir, describir y especificar las funciones y responsabilidades relacionadas con el proceso de reintegro, y designar un responsable que asegure la interacción entre las distintas partes involucradas ${ }^{36}$. Respecto a las percepciones entre unos y otros actores durante el proceso, los trabajadores tienden a centrarse en sus sentimientos con respecto al trabajo, los empleadores en el potencial de rendimiento del trabajador una vez que retorna, y los tratantes en la capacidad de adaptación al trabajo por parte del paciente, siendo más similares las visiones de estos dos últimos, respecto a la visión del trabajador ${ }^{18}$.

c) Aspectos relacionados con el tratamiento

El tratamiento es un aspecto importante, aunque no determinante para que un trabajador pueda reincorporarse exitosamente a su trabajo. En particular, la evidencia destaca el desarrollo de intervenciones con una perspectiva multidisciplinaria para comprender y abordar la patología ${ }^{34,36}$. Se enfatiza la necesidad de que la intervención ten- ga una clara orientación al lugar de trabajo, y que exista la posibilidad real de desarrollar cambios en la situación laboral, permitiendo un manejo beneficioso del proceso de rehabilitación ${ }^{30}$. Muijzer ${ }^{19}$ plantea que una intervención temprana enfocada en el trabajador, en la reducción del estrés, el entrenamiento en habilidades, y el apoyo del supervisor en el trabajo, serán aspectos clave que promoverán una conducta positiva del trabajador para el reintegro temprano. Sin embargo, cuando el reposo es prolongado, será la predominancia de los factores psicosociales que rodean al trabajador, por sobre sus síntomas, los que influenciarán el reintegro. Asimismo, el diagnóstico clínico tiene una fuerte asociación con el proceso de reintegro y la duración total del reposo por enfermedad ${ }^{37}$. Por otro lado, una atención psiquiátrica especializada en el ámbito ocupacional disminuye el tiempo de retorno laboral al compararse con la atención habitual ${ }^{38}$.

Cuando existe un diagnóstico de depresión, el proceso de reintegro laboral tendrá particularidades debido a los sentimientos de culpa, vergüenza, dificultad para concentrarse y las dificultades para evaluar desde una perspectiva más amplia las situaciones prácticas e interpersonales. En este sentido, el tratamiento no debiese enfocarse sólo en los aspectos laborales que produjeron o desencadenaron la patología, sino también en promover el apoyo al paciente (laboral, socialfamiliar) y procurar el estímulo de experiencias positivas en el paciente. Junto con ello, el supervisor debiera tener especial cuidado en los síntomas que impiden una adecuada funcionalidad laboral. A mayor claridad de las dificultades del paciente, mejor capacidad para hacer los ajustes al puesto de trabajo en relación a las necesidades y capacidades de éste ${ }^{18}$.

Respecto al tipo de técnica psicoterapéutica aplicada, aquellas que se sustentan en una terapéutica cognitivo-conductual centrada en el trabajo (TCC-T) han sido descritas como más efectivas para el reintegro al compararse con el tratamiento habitual ${ }^{39}$. Para considerar una intervención terapéutica como exitosa -en la que el proceso de reintegro laboral sea rápido y estable en el tiempo-, se señala como importante: a) la identificación temprana del equipo que liderará el proceso de reintegro; b) una evaluación multidisciplinaria con una aproximación holística; c) el diseño de un plan de tratamiento ajustado al 
paciente, en el que los objetivos se enfoquen en los obstáculos y recursos del mismo; d) una adecuada coordinación del equipo de reintegro, para que el proceso sea coherente y e) ajustes periódicos al tratamiento, para otorgar flexibilidad al proceso de cambio del paciente ${ }^{29}$.

\section{Discusión}

El reintegro laboral de un trabajador con patología mental es un proceso complejo, caracterizado por la necesidad de coordinación entre empleador, empleado y tratante para que sea exitoso. Es posible caracterizar diversas posturas respecto al trabajador, el ambiente de trabajo y el tratamiento recibido. Ahora bien, una interpretación de segundo orden, enfocada en aquellos aspectos transversales, nos permite comprender más dinámicamente el proceso de reintegro desde las perspectivas de: a) la temporalidad del reposo y las intervenciones; b) la calidad de la relación entre empleador y empleado durante el proceso, y c) la precisión y focalización del tratamiento para el reintegro laboral.

La temporalidad hace referencia a que a menor duración del reposo laboral, mayor será la probabilidad de un reintegro positivo al trabajo. Lo anterior se vincula con la necesidad de contacto entre empleador y trabajador durante el reposo. Si bien no existe consenso si este contacto debe implementarse desde el inicio del reposo, o si debe mantenerse de manera permanente durante la ausencia del trabajador, hay acuerdo en la necesidad de un contacto enfocado en el apoyo y preocupación por el estado de salud, y en la planificación del reintegro. Para que dicho reposo sea menor, el inicio del tratamiento deberá comenzar en las primeras seis semanas desde la primera consulta del trabajador. En esta misma línea, la temporalidad se relaciona con una intervención coordinada y especializada rápidamente en los síntomas que permitan una funcionalidad laboral, y en una descripción precisa de los problemas del paciente que posibilite hacer los ajustes al puesto de trabajo. En reposos de larga duración, atender los factores psicosociales que rodean al trabajador será más recomendable que focalizarse en su sintomatología, por lo que una intervención coordinada hacia el ambiente psicosocial y multidisciplinaria será lo deseable ${ }^{32}$.
El apoyo real y percibido de la jefatura directa del trabajador es un aspecto relacional que facilitará el reintegro al trabajo. La confianza del empleado y supervisor en las capacidades para realizar las tareas del cargo, además de informar cambios y mantener al día al trabajador en reposo serán aspectos esenciales ${ }^{12,15,39}$. Por otro lado, la modificación en las tareas que reduzcan la carga e intensidad de trabajo en un comienzo, o fortalecer el reintegro progresivo, se destacan como alternativas recomendables en este proceso.

Un diagnóstico clínico acucioso aplicado a las dificultades del trabajador para mantener su funcionalidad laboral es un aspecto esencial cuando nos referimos a la precisión y focalización de la intervención. Las diferencias por diagnóstico, edad, sexo, historia clínica y tiempo de reposo del trabajador son aspectos destacados en las investigaciones revisadas. La intervención enfocada en las particularidades del trabajador al enfrentar su puesto y ambiente de trabajo, el desarrollo de habilidades de afrontamiento, procurar experiencias positivas en el trabajo y de su entorno psicosocial serán recomendables para el éxito del proceso. Junto con ello se recomienda la planificación consciente y racional del reintegro, por parte del empleador.

La literatura internacional destaca acciones e intervenciones específicas que promueven un reintegro laboral exitoso en patologías mentales de origen laboral. Si bien la evidencia es contundente en países desarrollados, especialmente en Europa, no se encontraron publicaciones latinoamericanas que permitan representar nuestra realidad sociocultural. Es preciso señalar en este punto, la importancia de considerar un macronivel de intervención desde las políticas públicas y la protección social, puesto que los sistemas de seguridad social a nivel del país juegan un rol fundamental en la probabilidad de un reintegro laboral exitoso ${ }^{19}$.

En síntesis, lo que facilita el reintegro es la forma en que los involucrados comparten significados, se involucran en un objetivo común y construyen una forma propia de resolver los estresores identificados en el origen de la patología. El reintegro al trabajo será, entonces, un proceso de constante intercambio de información, expectativas y significados, junto con acuerdos a cumplir y a evaluar para avanzar en lo que resulta útil para empleado y empleador.

Para ampliar el conocimiento y efectividad de 
Facilitadores para el reintegro laboral en personas con patología mental - E. Ansoleaga et al

las intervenciones propuestas, se requieren futuros estudios que analicen procesos de reintegro laboral basados en la evidencia y comportamiento de la realidad local. Si bien no existe evidencia nacional publicada sobre este fenómeno, observamos una creciente preocupación por la salud mental ocupacional en distintos niveles. En el campo de la investigación, se observa un progresivo interés por estudiar la temática ${ }^{5,40-41}$.

El desarrollo del conocimiento local y regional en el ámbito del reintegro laboral resulta fundamental para avanzar en el desarrollo de políticas públicas que promuevan e incentiven a los actores de la tríada empleado-empleador-mutualidad a desarrollar acciones positivas que promuevan el reintegro al trabajo posterior a una enfermedad.

Agradecimientos: Agradecemos la colaboración recibida de la Clínica Psicológica y el Programa de Estudios Psicosociales del Trabajo, ambos de la Universidad Diego Portales, del Núcleo Milenio de Investigación en Depresión (Iniciativa Científica Milenio del Ministerio de Economía, Fomento y Turismo, Proyecto NS100018), de la Coordinación Nacional de Salud Mental de la Asociación Chilena de Seguridad, de su Fundación Científica y Tecnológica, responsable principal del financiamiento del proyecto, y de la Biblioteca de la Asociación Chilena de Seguridad por su generosa ayuda en la recopilación de los artículos que constituyen parte de esta revisión. A Marta Cozar por su valiosa y desinteresada revisión del texto.

\section{Referencias}

1. Andrade V, Carolina I. Salud Laboral. Investigaciones realizadas en Colombia. Pensamiento Psicológico; 4 (10): 9-25.

2. Garrido P, Ansoleaga M, Tomicic A, Domínguez C, Castillo S, Lucero C, et al. Afecciones de Salud Mental y el Proceso de Retorno al Trabajo: una Revisión Sistemática. Ciencia \& Trabajo 2013; (48): 105-13.

3. WHO-AIMS. Informe sobre los sistemas de salud mental en América Latina y El Caribe (2013); ISBN 978-92-75317332-4.

4. Ley 16.744. Seguridad Social. Establece Normas sobre Accidentes del Trabajo y Enfermedades Profesionales. Diario Oficial № 26957 Honorable Congreso aprobó el Proyecto de Ley.

5. Ansoleaga E, Montaño R, Vézina M. Psychosocial risk at work and hazardous alcohol consumption among
Chile's working adults. Canadian Journal of Public Health. Revue Canadienne de Santé Publique 2013; 104 (7): e502-8.

6. Fernandes F, Melo C, Gusmáo M, Fernandes J, Guimaráes A. Salud Mental y Trabajo: Significados y límites de modelos teóricos. Rev Latino-am Enfermagem 2006; 14 (5).

7. Ministerio de Salud. Protocolo de Vigilancia de los Riesgos Psicosociales del Trabajo. Departamento de Salud Ocupacional. División de Políticas Públicas Saludables y Promoción. Subsecretaria de Salud Pública. Ministerio de Salud-Chile 2012.

8. Identificación de los Factores de Riesgo Psicosocial en el Panorama General de Riesgos de la Empresa (Acuerdo 496/90; Resolución 2646/2008, Colombia), 2008.

9. Smetanin P, Stiff D, Briante C, Adair C, Ahmad S, Khan $\mathrm{M}$. The life and economic impact of major mental illnesses in Canada: 2011 to 2041. Risk Analytica, on behalf of the Mental Health Commission of Canada 2011. En: Mental Health Commission of Canada. Changing directions, changing lives: The mental health strategy for Canada 2012. Calgary, AB: Author.

10. Ansoleaga E, Valenzuela E. Salud Mental y Derechos Humanos: La salud de segunda categoría. En: Informe anual sobre Derechos Humanos en Chile 2013. Centro de Derechos Humanos UDP 2013. Ediciones Universidad Diego Portales.

11. Trucco B, Rebolledo M. Neurosis profesional o enfermedad común: Síntomas y estresores. Rev Med Chile [revista en la Internet] 2011 Oct [citado en $2014 \mathrm{Abr}$ 28]; 139 (10): 1370-7. Disponible en: http://www. scielo.cl/scielo.php?script=sci_arttext\&pid=S003498872011001000019\&lng=es. http://dx.doi.org/10.4067/ S0034-98872011001000019.

12. Taylor P. Mental Illness Costs the Canada Economy a Lot. The Globe and Mail Published; 2012, 19.

13. Cornelius LR, van der Klink JJ, Groothoff JW, Brouwer S. Prognostic factors of long term disability due to mental disorders: a systematic review. J occup rehabil 2011; 21 (2): 259-74.

14. Hoefsmit N, Houkes I, Nijhuis FJ. Intervention Characteristics that Facilitate Return to Work After Sickness Absence: A Systematic Literature Review. J occup rehabil 2012; 22: 462-77.

15. Kuoppala J, Lamminpaa A. Rehabilitation and work ability: a systematic literature review. J rehabil med 2008; 40 (10): 796-804.

16. Andersen M, Nielsen K, Brinkmann S. Meta-synthesis of qualitative research on return to work among employees with common mental disorders. Scandinavian Journal of Work, Environment \& Health 2012; 38 (2): 93-104. 
Facilitadores para el reintegro laboral en personas con patología mental - E. Ansoleaga et al

17. Urrutia G, Bonfill X. Declaración PRISMA: una propuesta para mejorar la publicación de revisiones sistemáticas y metanálisis. Med Clin 2010; 135 (11): 507-11.

18. De Vries GG, Koeter MJ, Nabitz UU, Hees HL, Schene AH. Return to work after sick leave due to depression: A conceptual analysis based on perspectives of patients, supervisors and occupational physicians. Journal of Affective Disorders 2012; 136 (3): 1017-26.

19. Muijzer A, Brouwer S, Geertzen JH, Groothoff JW. Exploring factors relevant in the assessment of the returnto-work process of employees on long-term sickness absence due to a depressive disorder: a focus group study. BMC Public Health 2012; 12: 103-10.

20. D'Amato A, Zijlstra F. Toward a climate for work resumption: the nonmedical determinants of return to work. Journal of occupational and environmental medicine 2010; 52: 67-80.

21. Brouwers E, Terluin B, Tiemens B, Verhaak P. Predicting return to work in employees sick-listed due to minor mental disorders. Journal of Occupational Rehabilitation 2009; 19 (4): 323-32.

22. Nielsen MB, Madsen IE, Bultmann U, Christensen U, Diderichsen F, Rugulies R. Predictors of return to work in employees sick-listed with mental health problems: findings from a longitudinal study. European journal of public health 2011; 21 (6): 806-11.

23. Clausen T, Friis M, Christensen K, Lund T. Return to work among employees with long-term sickness absence in eldercare: a prospective analysis of register-based outcomes. International Journal of Rehabilitation Research 2011; 34 (3): 249-54.

24. Brouwer S, Reneman M, Bültman U, Van der Klink J, Groothoff J. A prospective study of return to work across health conditions: perceived work attitude, self-efficacy and perceived social support. Journal of occupational rehabilitation 2010; 20; 104-12.

25. Lander F, Friche C, Tornemand H, Andersen J, Kirkeskov L. Can we enhance the ability to return to work among workers with stress-related disorders? BMC Public Health 2009; 9: 372-80.

26. Hees H, Nieuwenhuijsen K, Koeter M, Bultmann U, Schene AH. Towards a new definition of return-to-work outcomes in common mental disorders from a multistakeholder perspective. PLoS One 2012; 7 (6): 39947.

27. Roelen C, Norder G, Koopmans P, van Rhenen W, van der Klink J, Bültmann U. Employees sick-listed with mental disorders: who returns to work and when? Journal of occupational rehabilitation 2012; 22 (3): 409-17.

28. De Rijk A, Janssen N, Alexanderson K, Nijhuis F. Gender differences in return to work patterns among sickness absentees and their associations with health: a pros- pective cohort study in The Netherlands. International journal of rehabilitation research 2008; 31 (4): 327-36.

29. Martin MH, Nielsen MB, Petersen SM, Jakobsen LM, Rugulies R. Implementation of a coordinated and tailored return-to-work intervention for employees with mental health problems. Journal of occupational rehabilitation 2012; 22 (3): 427-36.

30. Karlson B, Jönsson P, Pålsson B, Åbjörnsson G, Malmberg B, Larsson B, et al. Return to work after a workplace-oriented intervention for patients on sick-leave for burnout-a prospective controlled study. BMC Public Health 2010; 10: 301-10.

31. Lemieux P, Durand M, Hong Q. Supervisors' perception of the factors influencing the return to work of workers with common mental disorders. Journal of Occupational Rehabilitation 2011; 21 (3): 293-303.

32. Tjulin $\AA$, Maceachen E, Ekberg K. Exploring the meaning of early contact in return-to-work from workplace actors' perspective. Disability \& Rehabilitation 2011; 33 (2): $137-45$

33. Muijzer A, Groothoff JW, Geertzen JH, Brouwer S. Influence of Efforts of Employer and Employee. J Occup Rehabil 2011; 21 (4): 513-9.

34. Netterstrom B, Bech P. Effect of a multidisciplinary stress treatment programme on the return to work rate for persons with work-related stress. A non-randomized controlled study from a stress clinic. BMC Public Health 2010; 10: 658-66.

35. De Rijk A, Nijhuis F, Alexanderson K. Gender differences in work modifications and changed job characteristics during the return-to-work process: a prospective cohort study. Journal of occupational rehabilitation 2009; 19 (2): 185-93.

36. Wahlin C, Ekberg K, Persson J, Bernfort L, Öberg B. Association between clinical and work-related interventions and return-to-work for patients with musculoskeletal or mental disorders. Journal of rehabilitation medicine 2012; 44 (4): 355-62.

37. Flach P, Groothoff J, Krol B, Bültmann U. Factors associated with first return to work and sick leave durations in workers with common mental disorders. European journal of public health 2012; 22 (3): 440-5.

38. Van der Feltz-Cornelis C, Hoedeman R, Jong F, Meeuwissen J, Drewes H, Van der Laan N, et al. Faster return to work after psychiatric consultation for sicklisted employees with common mental disorders compared to care as usual. A randomized clinical trial. Neuropsychiatric disease and treatment 2010; 6: 375-85.

39. Lagerveld SE, Blonk RW, Brenninkmeijer V, Wijngaardsde Meij L, Schaufeli WB. Work-focused treatment of common mental disorders and return to work: a com- 
Facilitadores para el reintegro laboral en personas con patología mental - E. Ansoleaga et al

parative outcome study. J occup healthpsychol 2012; 52 (1): 67-80.

40. Reavley NJ, Ross A, Killackey EJ, Jorm AF. Development of guidelines to assist organisations to support employees returning to work after an episode of anxiety, depression or a related disorder: a Delphi consensus study with Australian professionals and consumers. BMC Psychiatry 2012; 12 (1): 135.
41. Ansoleaga E, Vezina M, Montano R. [Depressive symptoms and work-related stress in Chilean workers: differential conditions for males and females]. Cadernos de saudepublica 2014; 30 (1): 107-18. Epub 2014/03/15.

42. Ansoleaga E, Montaño R, Vézina M. Validation of two complementary instruments for measuring work stress in Chilean workers. Scandinavian Journal of Organizational Psychology 2013; 5 (2): 5-14. 